\title{
Modulation of excitatory synaptic coupling facilitates synchronization and complex dynamics in a nonlinear model of neuronal dynamics
}

\author{
Michael Breakspear ${ }^{\mathrm{a}, *}$, John R. Terry ${ }^{\mathrm{b}}$, Karl J. Friston ${ }^{\mathrm{c}}$ \\ ${ }^{a}$ School of Physics and Brain Dynamics Centre, University of Sydney, 11A Wilson Street, Maroubra, \\ NSW 2035, Australia \\ ${ }^{\mathrm{b}}$ Department of Mathematics, University of Loughborough, Loughborough, UK \\ ${ }^{\mathrm{c}}$ Wellcome Department of Imaging Neuroscience, University College London, London, UK
}

\begin{abstract}
We study dynamical synchronization in a model of a neural system constituted by local networks of densely interconnected excitatory and inhibitory neurons. Neural dynamics are determined by voltage- and ligand-gated ion channels. Coupling between the local networks is introduced via sparse excitatory connectivity. With modulation of this long-range synaptic coupling the system undergoes a transition from independent oscillations to chaotic synchronization. Between these states exists a 'weakly' stable state with epochs of synchronization and complex intermittent desynchronization. This may facilitate adaptive brain function by engendering a diverse repertoire of dynamics and contribute to the genesis of complexity in the EEG.

(C) 2003 Elsevier Science B.V. All rights reserved.
\end{abstract}

Keywords: Synchronization; Intermittency; Neuromodulation

\section{Introduction}

The mechanisms and function of cooperative behavior in large-scale neuronal systems is a very active area of research. Synchronous oscillations between neurons have been proposed as a mechanism for perceptual 'binding' through the coupling of distinct neuronal populations to form dynamic cell assemblies, each encoding various aspects

\footnotetext{
* Corresponding author. Fax: +61-2-9351-7726.

E-mail address: mbreak@physics.usyd.edu.au (M. Breakspear).
} 
of a perceived object [11]. Evidence of coherent oscillations has been demonstrated in neurophysiological data [4] and computational models of the cortex [7]. However, in much of this research, only linear measures of synergistic activity are employed. The nonlinear properties of excitable membranes motivates the study of nonlinear aspects of synchronicity in neural systems. In this paper, we study nonlinear interdependence in a model neural system consisting of an array of coupled small-scale neural subsystems. The types and stability of nonlinear synchronization are investigated and the possible role of these behaviors in adaptive brain function is briefly discussed.

\section{Evolution equations}

The approach adopted in this study is to consider the behavior of local ensembles of neurons, with dynamical variables taken as ensemble averages. The scale of each ensemble is taken as the extension of pyramidal cell dendritic arbors, approximately $300 \mu \mathrm{m}$ (the size of cortical columns). The dynamical variables studied are the mean membrane potential of pyramidal cells, $V$, and inhibitory interneurons, $Z$, and the average number of 'open' potassium ion channels, $W$. The evolution equations are adapted from a study of epileptic seizures in hippocampal slices [6] consisting of coupled differential equations, which in turn are derived from the model of Morris and Lecar [8]. The mean cell membrane potential of the pyramidal cells is governed by the conductance of sodium, potassium and calcium ion through voltage- and ligand-gated membrane channels,

$$
\begin{aligned}
\frac{\mathrm{d} V}{\mathrm{~d} t}= & -\left(g_{\mathrm{Ca}}+r_{\mathrm{NMDA}} a_{\mathrm{ee}} Q_{V}\right) m_{\mathrm{Ca}}\left(V-V_{\mathrm{Ca}}\right)-\left(g_{\mathrm{na}} m_{\mathrm{na}}+a_{\mathrm{ee}} Q_{V}\right)\left(V-V_{\mathrm{na}}\right) \\
& -g_{K} W\left(V-V_{K}\right)-g_{\mathrm{L}}\left(V-V_{\mathrm{L}}\right)+a_{\mathrm{ie}} Z Q_{Z}+a_{\mathrm{ne}} I_{\delta}, \\
\frac{\mathrm{d} Z}{\mathrm{~d} t}= & b\left(a_{\mathrm{ni}} I_{\delta}+a_{\mathrm{ei}} V Q_{V}\right),
\end{aligned}
$$

where $g_{\text {ion }}$ is the maximum conductance of each population of ion species if all channels are open, $m_{\text {ion }}$ is the fraction of channels open, $V_{\text {ion }}$ is the Nernst potential for that ion species and $Q_{V(Z)}$ is the firing rate of the excitatory (inhibitory) neurons. The fraction of open channels is determined by the sigmoid-shaped 'neural activation function',

$$
m_{\text {ion }}=0.5\left(1+\tanh \left(\frac{V-V_{T}}{\delta_{\text {ion }}}\right)\right),
$$

where $\delta_{\text {ion }}$ incorporates the variance of this distribution. The fraction of open potassium channels is slightly more complicated, being governed by $W$, with

$$
\frac{\mathrm{d} W}{\mathrm{~d} t}=\frac{\phi\left(m_{k}-W\right)}{\tau},
$$


where $\phi$ is a temperature scaling factor and $\tau$ is a 'relaxation' time constant. Cell firing rate is also determined by sigmoid activation functions,

$$
Q_{V}=0.5 Q_{V \max }\left(1+\tanh \left(\frac{V-V_{T}}{\delta_{V}}\right)\right),
$$

where the $Q_{\max }$ are the maximum firing rate. An analogous term is also introduced for the inhibitory cells. The firing of these cell populations feeds back onto the ensemble through synaptic coupling to open ligand-gated channels and raise or lower the membrane potential accordingly. In the case of excitatory-to-inhibitory and inhibitory-toexcitatory connections, this is modeled as additional inputs to the flow of ions across the membrane channel, weighted by functional synaptic factors, $a_{\mathrm{ei}}$ and $a_{\mathrm{ie}}$. In the case of excitatory to excitatory connections, the rate of firing $Q_{V}$, is assumed to lead to a proportional release of glutamate neurotransmitter across the synapse, onto two classes of ligand-gated ion channels: (1) AMPA channels, which open an additional population of sodium channels, and (2) NMDA receptors, which open an additional population of voltage-gated sodium and calcium channels. $r_{\mathrm{NMDA}}$ incorporates the ratio of NMDA to AMPA receptors.

Each of the set of Eqs. (1)-(5) govern the dynamics within each local cell assembly. Coupling between $N$ nodes is introduced as competitive agonist excitatory action at the same populations of NMDA and AMPA receptors. Representing each node with a superscript, this is incorporated by modifying Eq. (1) to

$$
\begin{aligned}
\frac{\mathrm{d} V^{i}}{\mathrm{~d} t}= & -\left(g_{\mathrm{Ca}}+(1-C) r_{\mathrm{NMDA}} a_{\mathrm{ee}} Q_{V}^{i}+C r_{\mathrm{NMDA}} a_{\mathrm{ee}}\left\langle Q_{V}\right\rangle\right) m_{\mathrm{Ca}}\left(V^{i}-V_{\mathrm{Ca}}\right) \\
& -g_{K} W\left(V^{i}-V_{K}\right)-g_{\mathrm{L}}\left(V^{i}-V_{\mathrm{L}}\right)-\left(g_{\mathrm{na}} m_{\mathrm{na}}+(1-C) a_{\mathrm{ee}} Q_{V}^{i}\right. \\
& \left.+C a_{\mathrm{ee}}\left\langle Q_{V}\right\rangle\right)\left(V^{i}-V_{\mathrm{na}}\right)+a_{\mathrm{ie}} Z Q_{Z}^{i}+a_{\mathrm{ne}} I_{\delta}
\end{aligned}
$$

for $i, j=1, \ldots, N$. $C$ parameterizes the strength of excitatory coupling between cortical columns. If $C=0$ the systems evolve independently. $C>0$ introduces interdependences between consecutive columns. $C=1$ corresponds to maximum coupling, with excitatory input from outside each column surpassing excitatory input from within each column.

All physiologically measurable parameters (conductances, threshold potentials and Nernst potentials) are set to their accepted values [6], These are given in Table 1.

\begin{tabular}{ll}
$\begin{array}{l}\text { Table } 1 \\
\text { Parameter values for the simulations pre- } \\
\text { sented in this paper }\end{array}$ \\
\hline$I$ & 0.3 \\
$a_{\mathrm{ee}}$ & 0.4 \\
$a_{\mathrm{ei}}$ & 0.1 \\
$a_{\mathrm{ie}}$ & 1 \\
$a_{\mathrm{ne}}$ & 1 \\
$a_{\mathrm{ni}}$ & 0.4 \\
$r_{\mathrm{NMDA}}$ & 0.2 \\
$\delta$ & 0.001 \\
\end{tabular}




\section{Transition to chaotic synchronization}

With no intercolumn coupling $(C=0)$, each of the systems exhibit aperiodic asynchronous oscillations. Using a Gram-Schmidt orthonormalization procedure, we find that the largest Lyapunov exponent for each system is approximately 0.018 , indicative of chaotic motion. As expected, further exponents were identically zero and negative.

With the introduction of coupling, $C>0$, the systems show epochs of synchronized behavior. At $C \cong 0.1$, there is a transition to fully synchronized motion, as evident in the time series, Fig. 1a where $C=0.13$. Fig. $1 \mathrm{~b}$ shows the concurrent values of $V^{1}$ and $V^{2}$, for a system that have become embedded in the low-dimensional 'symmetry manifold' $V^{1}=V^{2}$. In addition to calculating the Lyapunov exponents for each system it is also possible to calculate the transverse Lyapunov exponents, which describe the rate of separation of the coupled systems transverse to this manifold-in other words, the time-average tendency for the two systems to synchronize or separate. For this strength of coupling, the largest transverse exponent is negative $(-0.005)$, indicating that perturbations away from this state of identical synchronization decay and thus that the state of identical chaotic synchronization is stable to noise. In Fig. 1(c) we present the value of the 4 largest Lyapunov exponents. It can be seen that the largest transverse Lyapunov exponent $\left(\lambda_{1}\right)$ crosses zero at $C \cong 0.1$. This represents the value at which a 'blowout' bifurcation occurs - so called because the dynamics will 'blow out' from the three-dimensional symmetry manifold into the full six dimensions [9].

We note two other observations. Increasing $r_{\mathrm{NMDA}}$, which corresponds to increasing the population of NMDA receptors, decreases the coupling strength required to achieve stable synchronization. On the other hand, increasing the overall excitatory-to-excitatory synaptic connectivity $\left(a_{\mathrm{ee}}\right)$ has the opposite effect. This is because the effect of local excitatory feedback, which tends to increase the local Lyapunov exponents, has the effect of 'splitting' the synchronization, over-riding the opposing effect of increasing inter-columnar coupling.

\section{Chaotic phase synchronization}

In the above simulations, all the parameters of both systems were equal. In real physiological systems, such symmetry between systems is clearly impossible. To incorporate this physiological variability, we introduced a random mismatch of between $0 \%$ and $10 \%$ for each of the physiological parameters. When the symmetry is broken, the symmetry manifold is no longer an invariant of the governing equations and hence identical synchronization between the coupled systems cannot be achieved. However, other types of synchronization are possible. In this section, we briefly illustrate an example of phase synchronization between subsystems in the neural model.

In Fig. 2(a) the time series for an example simulation with $C=0.2$ is shown. It can be seen that although the systems are not identical, the systems are not oscillating independently. Close inspection reveals that the systems are phase-locked, half a cycle out of phase. In panel (b), the concurrent values of $V^{1}$ and $V^{2}$ are plotted. It can be seen that although the systems are not identical, their evolution is contained on 

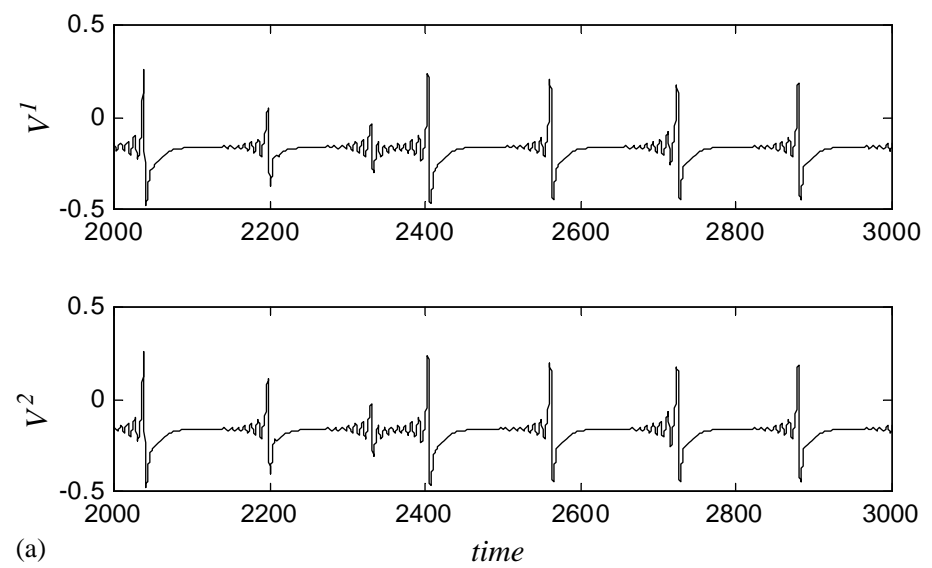

(a)

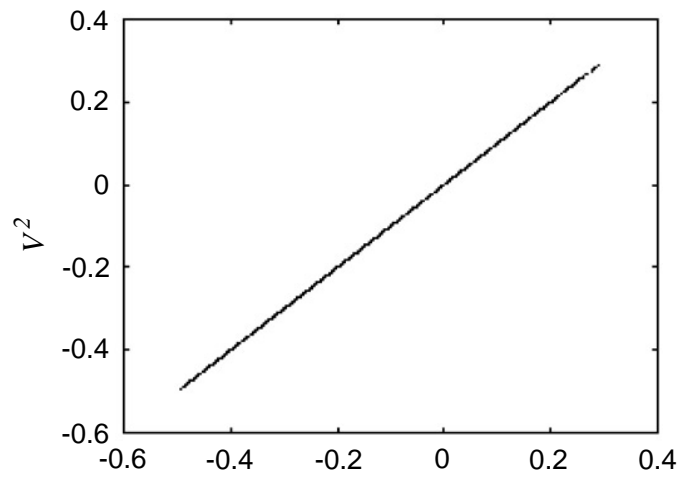

(b)

$$
V^{l}
$$

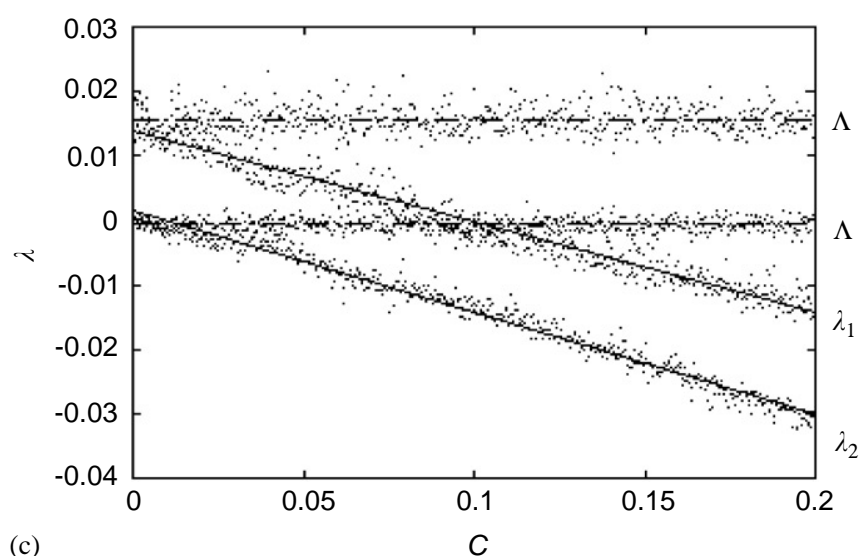

Fig. 1. (a) Time series of identical chaotic synchronization. (b) Concurrent values of $V^{1}$ and $V^{2}$. (c) Largest four Lyapunov exponents $\left(\lambda_{1}\right.$ and $\lambda_{2}$ are the transverse exponents). Note the zero crossing at $C=0.1$. 

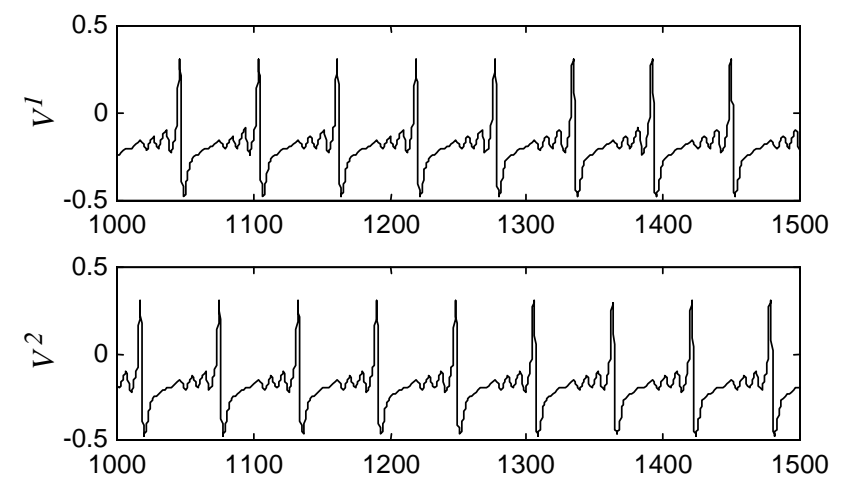

(a)

time

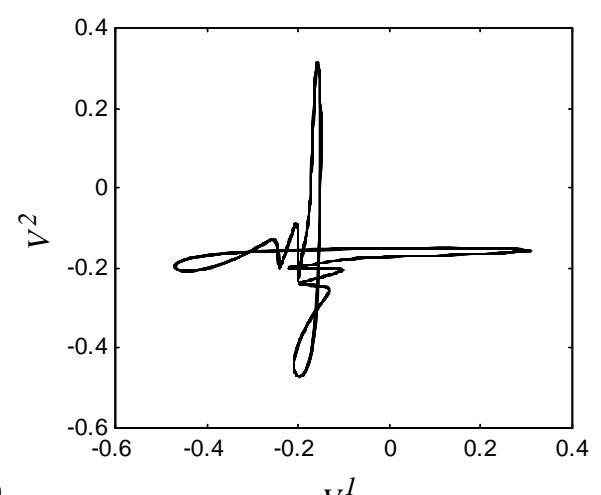

(b)

Fig. 2. (a) Time series of chaotic phase synchronization. (b) Concurrent values of $V^{1}$ and $V^{2}$.

a highly structured low-dimensional 'phase synchronization' manifold. It is possible to better quantify this aperiodic phase synchronization with the use of the Hilbert transform [10].

\section{Marginal stability and chaotic transients}

When the coupling strength is set close to the threshold for stable chaotic synchronization (i.e. $\lambda_{1} \sim 0$ ) one observes epochs of varying length interrupted by bursts of desynchronization. An example of such a phenomenon is given in Fig. 3. At time 1000 , it can be seen that the two time series briefly diverge from phase synchronization. Whilst space constraints prohibit further illustration, use of the Hilbert transform revealed two complete phase desynchronizations at this instance.

Such desynchronous bursts are known to be caused by transversely unstable periodic orbits embedded within the synchronized chaotic attractor. Desycnhronization occurs whenever the system passes close to such an unstable orbit [5]. We have previously 

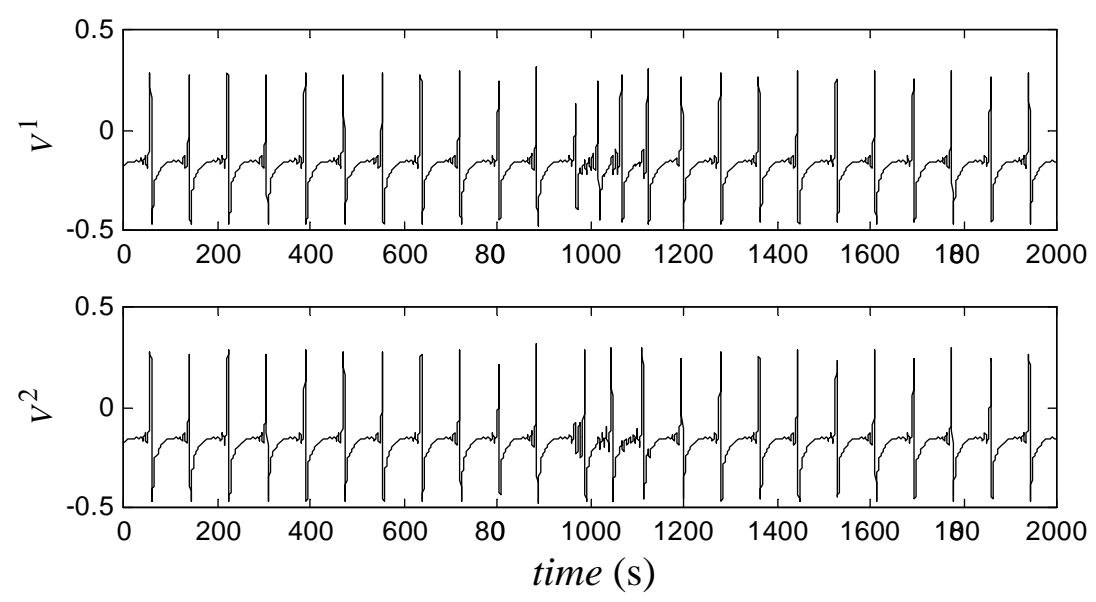

Fig. 3. Time series of weakly coupled system, showing brief desynchronization at $t \sim 1000 \mathrm{~s}$.

argued [1] that such 'marginal stability' may play a crucial role in normal brain function, permitting flexible and adaptive jumps between regional coherent states.

Analysis of larger arrays $(N>2)$ reveals that at judicious coupling strengths, the array breaks up into separate synchronized clusters of various sizes. In addition, it is possible to 'induce' synchronization between selected subsystems by increasing the coupling strength between them. This allows an extra level of complexity to be introduced into the model. For example, it may allow targeted information transfer between a partial network of the entire array.

\section{Discussion}

In this paper, we present a dynamical model for activity in an array of sparsely coupled local neural systems. The model incorporates the fundamental principles governing cell membrane potential, as determined by voltage and ligand-gated ion channels, and basic principles of cortical connectivity. In this way, it allows a reasonable amount of physiological complexity to be incorporated, including synaptic activation of receptor subtypes. Numerical analysis of this model reveals a transition from independent oscillations to stable chaotic synchronization, depending on the balance of local versus long-range connectivity. These functional excitatory couplings are subject to subcortical neuromodulation. Chaotic phase synchronization occurs and is robust to system noise and random parameter variations.

Behaviors observed in the regime of 'marginal stability'-i.e. when the transverse Lyapunov exponent is close to zero - are of particular interest, as they are characterized by a dynamic balance between synchronous and desynchronous dynamics in both spatial and temporal domains. Such phenomenon may facilitate the formation and dissolution of separate clusters of dynamic cell assemblies, permitting optimal and flexible 
adaptation to a changing external environment [3]. Whilst speculative, this conjecture is supported by the finding of occasional brief instances of nonlinear interdependence in human scalp EEG data [1,2]. Further empirical investigations are required in order to determine the cognitive correlates of these phenomena.

\section{References}

[1] M. Breakspear, Hum. Brain Mapping 5 (2002) 175-198.

[2] M. Breakspear, J. Terry, Clin. Neurophysiol. 113 (2002) 735-753.

[3] K. Friston, Philos. Trans. Roy. Soc. London B 355 (2000) 237-252.

[4] C.Gray, P. Konig, A. Engel, W. Singer, Science 338 (1989) 334-337.

[5] J. Heagy, T. Carroll, L. Pecora, Phys. Rev. E 52 (1998) R1253-R1256.

[6] R. Larter, B. Speelman, Chaos 9 (1999) 795-804.

[7] E. Lumer, G. Edelman, G. Tononi, Cereb. Cortex 7 (1997) 207-227.

[8] C. Morris, H. Lecar, Biophys. J. 35 (1981) 193-213.

[9] E. Ott, J. Sommerer, Phys. Lett. A 188 (1994) 39-47.

[10] M. Rosenblum, A. Pikovsky, J. Kurths, Phys. Rev. Lett. 76 (1996) 1804-1807.

[11] W. Singer, Putative functions of temporal correlations in neocortical processing, in: C. Koch, J. Davis (Eds.), Large-Scale Neuronal Theories of the Brain, MIT Press, London, 1995. 